\title{
Emulsifiçação/gelificação iônica interna: Alternativa para microencapsulação de compostos bioativos
}

\author{
Emulsification/internal ionic gelation: Alternative for microencapsulation bioactive \\ compounds
}

\author{
Augusto Tasch Holkem ${ }^{1}$, Cristiane Franco Codevilla e Cristiano Ragagnin de Menezes $^{3}$ \\ ${ }^{1}$ Mestrando em Ciência e Tecnologia dos Alimentos, Departamento de Tecnologia e Ciência de Alimentos, \\ Universidade Federal de Santa Maria, Santa Maria, Brasil \\ augusto.holkem@hotmail.com \\ ${ }^{2}$ Pós-doutoranda em Ciência e Tecnologia dos Alimentos, Departamento de Tecnologia e Ciência de Alimentos, \\ Universidade Federal de Santa Maria, Santa Maria, Brasil \\ cristianefc@hotmail.com \\ ${ }^{3}$ Professor Adjunto do Centro de Ciências Rurais da Universidade Federal de Santa Maria, Departamento de \\ Tecnologia e Ciência de Alimentos, Universidade Federal de Santa Maria, Santa Maria, Brasil \\ cristiano.ufsm@gmail.com
}

\begin{abstract}
Resumo
A aplicação de compostos bioativos em alimentos tem atraído o interesse das indústrias, devido aos benefícios potencias para a saúde dos seres humanos. No entanto, a eficácia depende da estabilidade, bioatividade e biodisponibilidade destes ingredientes ativos na matriz alimentícia. O gosto desagradável e a instabilidade limitam aplicação destes compostos. A utilização de bioativos encapsulados, em vez de compostos livres, pode efetivamente amenizar estes problemas. A tecnologia de encapsulamento que está sendo utilizada em diversos compostos ativos é a emulsificação/gelificação iônica interna, que é uma alternativa importante na melhoria da estabilidade e aplicação em alimentos.
\end{abstract}

Palavras-chave: Bioativos. Emulsificação. Mcroencapsulação. Estabilidade.

\begin{abstract}
The application of bioactive compounds in food have attracted the interest of the industry due to potential health benefits to humans. However, the efficiency depends on the stability, bioactivity and bioavailability of these active ingredients in the food matrix. The unpleasant taste and instability limit the application of these compounds. The use of encapsulated bioactive instead of free compounds can effectively alleviate these problems. The encapsulation technology being used in many different active compounds is the emulsification/internal ionic gelation, which is an important alternative for improving the stability and application in food.
\end{abstract}

Keywords: Bioactive. Emulsification. Microencapsulation. Stability. 


\section{Introdução}

Os compostos bioativos são substâncias químicas naturais em alimentos que fornecem benefícios à saúde além do valor nutricional básico. Os principais bioativos são os carotenóides, fitoesteróis, flavonoides, fosfolipídeos, organosulfurados e os polifenóis (MUNIN \& EDWARDS-LÉVY, 2011; VOS et al., 2010). Eles são constituintes que normalmente estão presentes em pequenas quantidades em alimentos, mas estão sendo extensivamente estudados para avaliar os seus efeitos (BIESALSKI et al., 2009). O consumo frequente destes compostos através das frutas e vegetais tem sido associado a um menor risco de desenvolvimento de doenças, tais como acidente vascular cerebral, diabetes mellitus, artrite, Parkinson, Alzheimer e câncer (CROWE et al., 2011; KRIS-ETHERTON et al., 2002).

Nos últimos anos, tem ocorrido um crescimento significativo na procura de alimentos funcionais devido a mudança de estilo de vida da população. Atualmente, é um grande interesse de incorporar compostos bioativos em alimentos e bebidas devido aos seus efeitos benéficos sobre a saúde. No entanto, a maioria dos compostos bioativos apresentam elevada instabilidade ou sabor indesejável que impede muitas vezes a sua aplicação direta em alimentos. Além disso, o composto de interesse deve ser liberado a partir das micropartículas pós ingestão, sendo bioacessível no trato gastrointestinal até atingir o tecido alvo (CRUZ et al., 2015; ESPÍN et al., 2007; McCLEMENTS, 2014).

A encapsulação vem como alternativa para a proteção destes compostos contra o estresse ambiental e a degradação química, além de aumentar a estabilidade e a biodisponibilidade durante um período. Porém, a grande preocupação é a compatibilidade das técnicas de microencapsulação com a natureza do bioativo e a liberação do composto até seu local de ação. Entre as várias técnicas disponíveis para encapsulação, a emulsificação é um método eficaz para aumentar a absorção in vitro e in vivo dos bioativos. Muitos estudos relatam a grande possibilidade de microencapsular compo: hidrofílicos e hidrofóbicos através desta técı (CHAKRABORTY et al., 2009; EL-ABBASS al., 2013; McCLEMENTS, 2010).

A produção de micropartículas através técnica de emulsificação/gelificação iô1 interna é um método de encapsulação ráp que possui formulação e procedimento simץ de baixo custo e têm sido usualm empregado. Baseada na capacidade polieletrólitos de fazerem ligações cruzadas presença de contra-íons, formando uma má tridimensional. Por essa técnica, uma solu polimérica carregada negativamente fo] ligações cruzadas (cross-linking) com cátions baixa massa molecular, formando hidrogéis ( 2010; COSTA, 2014).

\section{Emulsificação/gelificação iônica interna}

$\mathrm{Na}$ gelificação iônica interna ocorre formação de uma emulsão que pode ser do 1 água em óleo (W/O), óleo/água (O/W) também óleo/óleo (O/O) (Figura 1). A adiçãc um emulsionante favorece a formação estabilidade da emulsão. Em ambos os caso fase chamada interna, onde o polímero encontra dissolvido, é um solvente orgânico apresenta uma solubilidade limitada na externa da emulsão, a qual pode ser áq formando uma emulsão. Um sal insolúvel parcialmente insolúvel de cálcio (normalmen carbonato de cálcio) já está presente no inte das gotículas da emulsão. Um ácido é er adicionado ao meio que se propaga ao longc fase contínua para as gotículas. Ocorre diminuição do $\mathrm{pH}$ e íons $\mathrm{Ca}^{+2}$ são liberadc sucede-se a gelificação aprisionando o compr ativo (PONCELET, 2001; O'DONNELL MCGINITY, 1997; CHAN et al., 2002). 


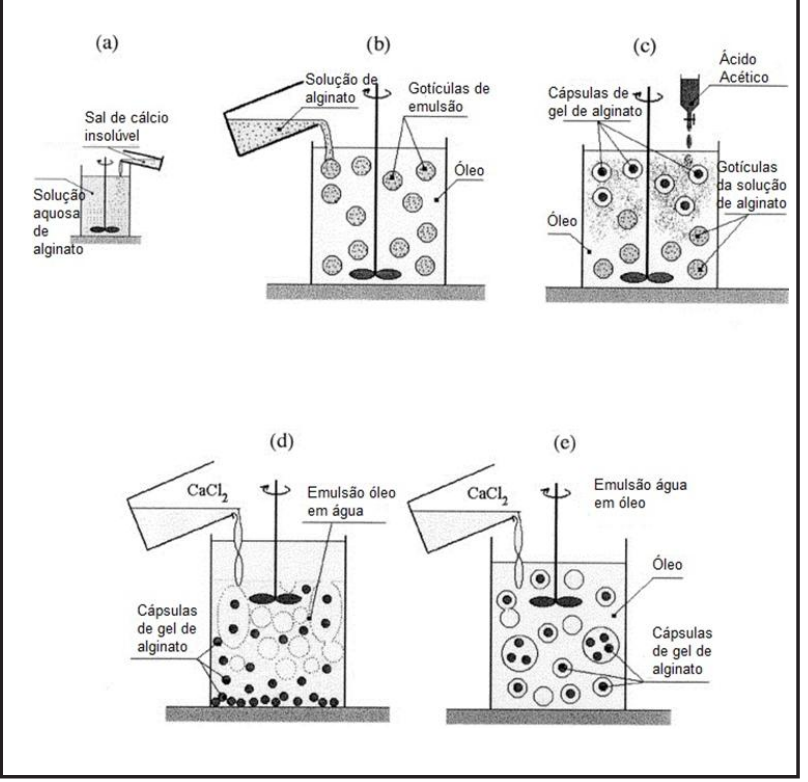

Figura 1 - Diferentes estágios de preparação de cápsulas de alginato pelo método de gelificação iônica interna (Adaptado de PONCELET et al., 1999)

Por meio da gelificação iônica, é possível a produção de dietas protéicas ricas em carboidratos, além de incorporar partículas insolúveis em água na sua composição com o uso de hidrocolóides. Atuando com eficiência como uma matriz impermeável para oxigênio, certos compostos orgânicos e acidez. As propriedades das partículas formadas pela gelificação como o volume, índice de difusão dos solutos para dentro ou fora da matriz, cinética de formação do gel e também a porosidade e estabilidade das cápsulas são influenciadas precisamente pela força iônica, concentração dos cátions e do polissacarídeo e pelo pH (MUKAICORRÊA et al., 2004; LUPO et al., 2014; PONCELET et al., 1992).

$\mathrm{Na}$ área de alimentos os hidrocolóides mais utilizados são o alginato e a pectina. São polieletrólitos naturais e com grande capacidade de formação de hidrogéis possuindo uma característica vantajosa de biocompatibilidade com membranas e ausência de tensão interfacial com fluidos (VOS et al., 2010; TEIXEIRA et. al., 2014; CHAN et al., 2006).

\section{Microencapsulação de polifenóis}

Os polifenóis são quimicamente estruturados como um grupo hidroxila ligado a um anel aromático. Estes compostos são metabólitos secundários sintetizados por plantas, variando de moléculas simples, tais como os ácidos fenólicos, para aqueles com muitos grupos fenólicos. Os polifenóis das frutas representam a principal fonte de antioxidantes para uso humano e tornaram-se um foco intenso de pesquisa devido aos seus efeitos benéficos para a saúde, especialmente no tratamento e prevenção do câncer e as doenças cardiovasculares e crônicas. O consumo médio de polifenóis é de cerca de $1 \mathrm{~g}$ por dia, sendo as principais fontes de frutas, chá e café, e em menor grau, legumes, cereais e leguminosas. (KAUR \& KAPOOR, 2001; NEVES, HASHEMI \& PRENTICE, 2015).

No estudo de Lupo et al. (2014) que microencapsularam extrato de polifenóis do cacau por emulsificação/gelificação iônica interna, observou-se que os sais de citrato e carbonato apresentou microcápsulas de diâmetro menor e uma eficiência de encapsulação de $60 \%$. As micropartículas de cacau poderia aumentar a ingestão diária de antioxidantes quando implementado em um produto alimentar.

Em outra pesquisa, com objetivo de imobilizar os compostos fenólicos de extratos de chá através da emulsificação realizaram a dissolução de tween 20 em água contendo uma infusão de chá liofilizado e albumina do soro bovino, o oléo de girassol (do qual os tocoferóis foram removidos) foi adicionado gota a gota e logo em seguida foi adicionado em banho de gelo e sonicado por 5 minutos. As emulsões W/O contendo extratos de chá mostraram forte atividade antioxidante contra a oxidação do óleo (ALMAJANO, CARBÓ, JIMÉNEZ \& GORDON, 2008).

\section{Microencapsulação de carotenóides}

Os carotenóides são tetraterpenóides de 40 carbonos unidos por unidades opostas no centro da molécula 1,7. Ciclização, hidrogenação, desidrogenação, encurtamento ou alongamento da cadeia, rearranjo, isomerização, introdução de funções com oxigênio ou a combinação destes processos resultam na diversidade de estruturas dos carotenóides. A capacidade dos carotenóides como agente quelante do oxigênio singlete molecular é bem conhecida. Assim como a prevenção do câncer, o potencial anti-oxidante dos carotenóides pode ser útil na inibição de outras doenças provocadas pela ação dos 
radicais livres (ROCK, 1997; RODRIGUESAMAYA, 1999; UENOJO, JUNIOR \& PASTORE, 2007).

Na pesquisa de Bustos et al. (2003) avaliaram microcápsulas de quitosana produzidas por emulsificação contendo ácidos graxos polinsaturados (PUFAs) e astaxantina como pigmento. As microcápsulas permaneceram estáveis e homogêneas, os tratamentos com quitosana de baixo peso molecular mostraram um melhor efeito protetor do que a quitosana de alto peso molecular. Existe um grande potencial de aplicação desse sistema de micropartículas para PUFAs e suplementação de vitamina lipossolúvel em alimentos.

O estudo de Shi et al. (2015) microencapsularam licopeno por emulsificações com duas camadas em isolado proteíco do soro do leite (WPC) e éster metílico de alta-pectina (HMP). A microemulsão de duas camadas que consistiu de WPC e HMP era muito mais estável sob estresse ambiental em comparação com o tratamento com uma camada de WPC. A eficiência de encapsulação foi de $89 \%$ para o tratamento com duas camadas, o que garante uma alta eficiência e estabilidade nesta técnica para estes compostos.

\section{Microencapsulação de óleos essenciais}

A microencapsulação de ácidos graxos essenciais pode ser realizada de diversas maneiras como podemos citar:

- Aprisionamento em matrizes vítreas

- Encapsulação em lipossomas

- Encapsulação em ciclodextrinas

Mas a maioria das técnicas envolvem a emulsificação como um passo fundamental. Diferentes tipos de sistemas baseados em emulsão utilizados para encapsular vários ingredientes lipofílicos na indústria alimentar incluem o óleo de camada única em emulsão de água, emulsões múltiplas e óleo em emulsão de água com camadas múltiplas (KAUSHIK, DOWLING, BARROW \& ADHIKARI, 2014). Proteção contra a deterioração oxidativa é o principal objetivo por trás da microencapsulação de óleos. A fim de alcançar o melhor nível de estabilidade da emulsão, formulações de emulsão diferentes foram tentadas usando uma variedade de ingredientes (KLINKESORN et al., 2005; OKURO et al., 2015).

No estudo de Laine et al. (2011) microencapsularam óleo da semente de colza através da emulsificação (óleo em água) utilizando farelo de aveia modificada e concluíram que entre as variáveis independentes, o conteúdo de farelo teve o maior efeito sobre as propriedades de emulsão, apesar da diminuição da pressão de homogeneização e da viscosidade. Farelo de aveia modificado possui uma excelente capacidade de formar emulsões estáveis que podem ser adequados para a microencapsulação.

$\mathrm{Na}$ pesquisa de Sarkar et al. (2012) que microencapsularam óleo de hortelã com goma arábica e goma guar (irradiadas) por emulsificação seguida por spray-dryer. As microcápsulas foram avaliadas quanto à retenção de óleo de hortelã durante o armazenamento de 8 semanas. Assim, a goma de guar irradiadas a 50 kGy poderia ser usado como substituição parcial de goma arábica para encapsulação de ingredientes alimentares sensíveis, pois nesta condição houve maior retenção durante as 8 semanas.

A emulsificação utilizando dupla emulsão está sendo muito empregado na encapsulação de óleos, pois proporciona uma melhor proteção para os ingredientes ativos (Figura 2). As emulsões podem ser de água-óleo-água ( $\mathrm{w}$ / o / w) ou óleo-água-óleo (o / w / o). Cho, Shim \& Park (2006) microencapsularam óleo de peixe em emulsões duplas feitas pela técnica de gelificação. Em primeiro lugar, uma emulsão de óleo-em-água foi produzida por dispersão de óleo de peixe em isolado de proteína de soja. Esta emulsão foi subsequentemente dispersa em óleo de milho para formar uma emulsão dupla o / w / o. A Enzima transglutaminase foi utilizada para iniciar a gelificação. As cápsulas assim obtidas eram esféricas com um diâmetro médio de $23 \mu \mathrm{m}$. Em geral, este método melhora a estabilidade dos ingredientes e permite a libertação controlada. 


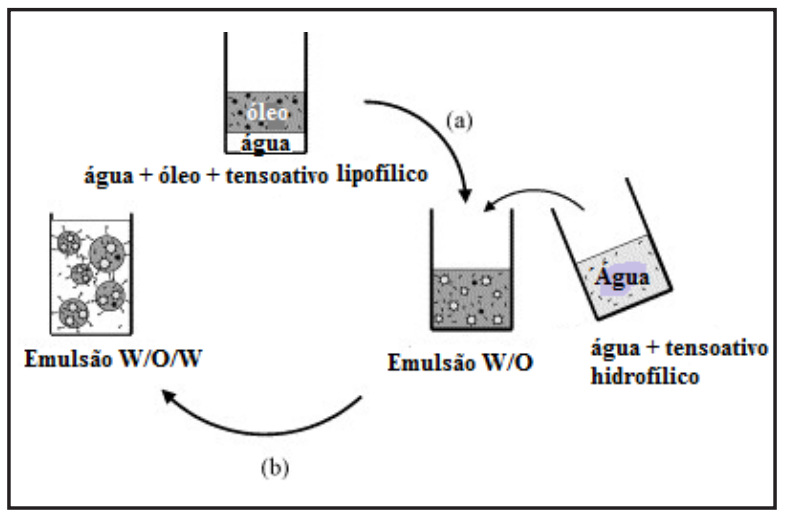

Figura 2 - Preparação de uma emulsão dupla W / $\mathrm{O} / \mathrm{W}$ em dois passos: primeiro passo a emulsificação de alto cisalhamento com tensoativos lipofílicos para a emulsão W / O (a) e segundo passo a emulsificação de baixo cisalhamento com tensoativos hidrofílicos para a emulsão W / O / W (b).

Fonte: Graaf, Schroën \& Boom (2005)

\section{Potencialidades do método para os bioativos}

As emulsões podem oferecer muitas vantagens para diversas aplicações, uma vez que se verificou ser uma estratégia potencialmente útil como:

- Produção de produtos de baixo teor calórico;

- Redução de gordura;

- Mascarar sabores;

- Evitar a oxidação;

- Melhorar as características sensoriais do alimento;

- Controlar a liberação e proteção ingredientes instáveis durante a alimentação e digestão.

A emulsificação tem inúmeras aplicações, como na área farmacêutica, na área médica e agronômica. No setor alimentício é algo promissor e muitos estudos estão sendo realizados (LAM \& GAMBARI, 2014). Como podemos citar abaixo:

Os edulcorantes que são substâncias de baixo valor de energia e fornecem sabor doce, mas não têm as calorias. A microencapsulação tem como objetivo de estabilizar e estender a libertação de doçura deste substituto do açúcar. Os estudos iniciais de Rocha-Selmi et al. (2013) microencapsularam aspartame por dupla emulsão seguida por coacervação complexa com objetivo de proteger e controlar sua liberação. As microcápsulas foram multinucleadas, pouco higroscópicas e mostrou taxas reduzidas de umidade. Os resultados indicaram que era possível encapsular o aspartame com as técnicas empregues e que este adoçante era protegido até mesmo a $80^{\circ} \mathrm{C}$. Os autores concluíram que a solubilidade reduzida e baixas taxas de liberação indicaram um enorme potencial para microencapsular o aspartame em alimentos, prolongando assim a sua doçura.

Um dos métodos mais eficientes de controle da liberação de enzimas é a emulsificação. $\mathrm{Na}$ pesquisa de Belyaeva, Valle \& Poncelet (2004) produziram micropartículas de carragena contendo $\alpha$ - quimotripsina por emulsificação. $\mathrm{O}$ tamanho das capsulas foi de $300 \mu \mathrm{m}$ sem perda de atividade por 2 semanas. $O$ que representa uma alta estabilidade e eficiência de encapsulação.

As pesquisas ainda são muito recentes com relação a microencapsulação de flavors por emulsificação. Essas emulsões diluídas são compostas de óleos de aroma, agente de ponderação para aumentar a densidade e agente emulsionante (goma arábica ou amido de milho modificado). Duas fases de homogeneização são empregadas para reduzir o tamanho das micropartículas. A estabilidade a longo prazo é o resultado de vários fatores interativos como tamanho de partícula, homogeneidade, densidade de equilíbrio entre a fase dispersa e a fase contínua e integridade química da fase dispersa. No estudo de Rabe, Krings \& Berger (2003) utilizaram emulsão o / w, os parâmetros de emulsão foram avaliados quanto à sua influência na liberação. $\mathrm{O}$ aroma foi liberado independente do tamanho das partículas da emulsão, e surpreendentemente afetado pelo tipo e concentração de emulsionante.

A tendência recente no consumo de alimentos resultou na substituição de gordura saturada por gorduras insaturadas na dieta. No entanto, as gorduras insaturadas são propensas a oxidação. Os compostos lipossolúveis naturais como o $\alpha$ tocoferol (vitamina E) pode ser usado como um antioxidante. No estudo de Sharipova et al. (2015) microencapsularam vitamina $\mathrm{E}$ através da emulsificação (óleo em água) logo em seguida foi aplicado uma técnica camada por camada para 
construir um revestimento oleoso. Concluíram que ocorreu uma desaceleração gradual e baixa liberação da vitamina foi observada em cada etapa da formação cápsulas. As micropartículas finais mostraram uma liberação controlada dos bioativos dentro de, pelo menos, 80 horas.

As emulsões podem ser aplicadas em escalada industrial por microcanais. Estudos tem desenvolvido grandes dispositivos para realizar a produção de gotículas de tamanho uniforme (Figura 3). O grande dispositivo de emulsificação tem uma produtividade superior a várias toneladas por ano, o que poderia satisfazer uma produção mínima em escala industrial de micropartículas e microcápsulas carregadas com compostos bioativos. Este sistema têm um potencial para aplicação em formulações de alimentos funcionais aumentando a biodisponibilidade destes compostos ativos (KHALID et al. 2014).

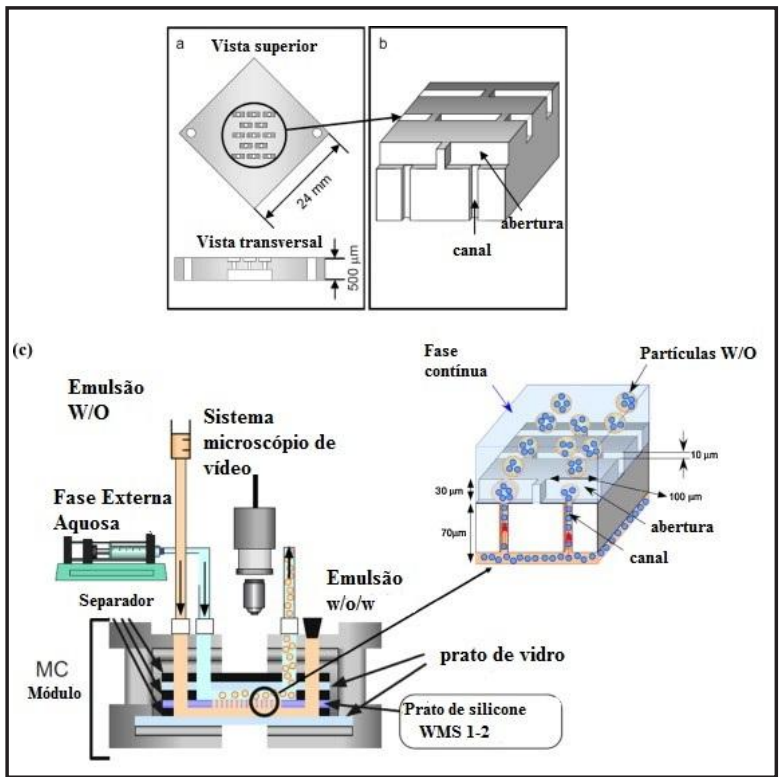

Figura 3 - Representações esquemáticas de (a) matriz MC assimétrica (WMS-1-2) placa, (b) assimétricos MCs, e (c) a instalação MCE e geração de W / O gotículas de emulsão. Fonte: Khalid et al. (2014)

\section{Conclusão}

A encapsulação proporciona um método eficaz para proteção um composto ativo oferecendo numerosas vantagens. É uma abordagem importante para atender todas as demandas, fornecendo componentes bioativos dos alimentos no momento certo e no lugar adequado. Uma possibilidade atraente é utilizar uma metodologia em que dois ou mais componentes bioativos podem ser combinadas para ter um efeito sinérgico. Isto vai desempenhar um papel significativo no aumento de alimentos funcionais nos próximos anos.

A emulsificação/gelificação iônica interna apresenta-se como um método eficiente para encapsulação de compostos sensíveis como os bioativos. A utilização de baixa concentrações de polissacarídeos e cloreto de cálcio resulta em uma estrutura interna desenvolvida e uma liberação controlada ao longo do tempo com diâmetros menores que $100 \mu \mathrm{m}$. A produção à frio e baixo custo de materiais faz desta técnica um potencial para emprego em escala industrial na encapsulação de ingredientes ativos.

\section{Referências}

ALMAJANO, M. P., CARBÓ, R., JIMÉNEZ, J. A. L., GORDON, M. H. Antioxidant and antimicrobial activities of tea infusions, Food Chemistry, v. 108, n. 1, p. 55-63, 2008.

BELYAEVA, E., VALLE, D. D., PONCELET, D. Immobilization of $\alpha$-chymotrypsin in $\kappa$ carrageenan beads prepared with the static mixer, Enzyme and Microbial Technology, v. 34, n. 2, p. 108-113, 2004.

BIESALSKI, H.-K., DRAGSTED, L.O., ELMADFA, I., GROSSKLAUS, R., MÜLLER, M., SCHRENK, D., WALTER, P., WEBER, P. Bioactive compounds: definition and assessment of activity, Nutrition, v. 25, n. 11-12, pp. 12021205, 2009.

BUSTOS, R., ROMO, L., YÁÑEZ, K., DÍAZ, G., ROMO, C. Oxidative stability of carotenoid pigments and polyunsaturated fatty acids in microparticulate diets containing krill oil for nutrition of marine fish larvae, Journal of Food Engineering, v. 56, n. 2-3, p. 289-293, 2003.

CHAKRABORTY, S., SHUKLA, D., MISHRA, B., SINGH, S. Lipid - an emerging platform for oral delivery of drugs with poor bioavailability, Eur. J. Pharm. Biopharm, v. 73, n. 1-15, 2009.

CHAN, L.W.; LEE, H.Y.; HENG, P.W.S. Production of alginate microspheres by internal gelation using an emulsification method, 
International Journal of Pharmaceutics, 242 (12), 259-262, 2002.

CHAN, L. W.; LEE, H. Y.; HENG, P. W.S. Mechanisms of external and internal gelation and their impact on the functions of alginate as a coat and delivery system, Carbohydrate Polymers, 63 (2), 176-187, 2006.

CHO, Y.H., SHIM, H.K., PARK, J. Encapsulation of fish oil by an enzymatic gelation process using transglutaminase cross-linked proteins, Journal of Food Science, v. 68, p. 2717-2723, 2006.

COSTA, B. S. Micropartículas produzidas por gelificação iônica recobertas com gelatina de peixe e isolado proteico de soja. Dissertação (Mestrado em alimentos e nutrição) Universidade Estadual de Campinas, CampinasSP, 2014.

CROWE, F.L., RODDAM, A.W., KEY, T.J., APPLEBY, P.N., OVERVAD, K., JAKOBSEN, M.U., et al. Fruit and vegetable intake and mortality from ischaemic heart disease: Results from the European prospective investigation into cancer and nutrition (EPIC)-heart study, European Heart Journal, v. 32, pp. 1235-1243, 2011.

CRUZ, Z., GARCÍA-ESTRADA, C., OLABARRIETA, I., RAINIERI, S. Chapter 16 Lipid Nanoparticles: Delivery System for Bioactive Food Compounds, In Microencapsulation and Microspheres for Food Applications, edited by Leonard M.C. Sagis, Academic Press, San Diego, p. 313-331, 2015.

EL-ABBASSI, A., NEVES, M.A., KOBAYASHI, I., HAFIDI, A., NAKAJIMA, M. Preparation and characterization of highly stable monodisperse argan oil-in-water emulsions using microchannel emulsification, Eur J Lipid Sci Technol, v. 115, pp. 224-231, 2013.

ESPÍN, J.C., GARCÍA-CONESA, M.T., TOMÁSBARBERÁN, F.A. Nutraceuticals: facts and fiction, Phytochemistry, 68, 2986-3008, 2007.

GRAAF, S. V. D., SCHROËN, C.G.P.H, BOOM, R.M. Preparation of double emulsions by membrane emulsification-a review, Journal of Membrane Science, v. 251, n. 1-2, pp. 7-15, 2005.

KAUR CH., KAPOOR, H.C. Antioxidants in fruits and vegetables - the millennium's health, International Journal of Food Science and Technology, 36, pp. 703-725, 2001.

KAUSHIK, P., DOWLING, K., BARROW, C. J., ADHIKARI, B. Microencapsulation of omega-3 fatty acids: A review of microencapsulation and characterization methods, Journal of Functional Foods, Available online 4 July 2014.

KHALID, N., KOBAYASHI, I., NEVES, M. A., UEMURA, K., NAKAJIMA, M., NABETANI, H. Monodisperse $\mathrm{W} / \mathrm{O} / \mathrm{W}$ emulsions encapsulating 1-ascorbic acid: Insights on their formulation using microchannel emulsification and stability studies, Colloids and Surfaces A: Physicochemical and Engineering Aspects, v. 458, pp. 69-77, 2014.

KLINKESORN, U., SOPHANODORA, P., CHINACHOTI, P., DECKER, E. A., MCCLEMENTS, D. J. Encapsulation of emulsified tuna oil in two-layered interfacial membranes prepared using electrostatic layerby-layer deposition, Food Hydrocolloids, v. 19, n. 6, p. 1044-1053, 2005.

KRIS-ETHERTON, P.M., HECKER, K.D., BONANOME, A., COVAL, S.M., BINKOSKI, A.E., HILPERT, K.F., GRIEL, A.E., ETHERTON, T.D. Bioactive compounds in foods: their role in the prevention of cardiovascular disease and cancer, Am. J. Med., v. 113, n. 9, pp. 71-88, 2002.

LAINE, P., TOPPINEN, E., KIVELÄ, R., TAAVITSAINEN, V., KNUUTILA, O., SONTAG-STROHM, T., JOUPPILA, KIRSI., LOPONEN, J. Emulsion preparation with modified oat bran: Optimization of the emulsification process for microencapsulation purposes, Journal of Food Engineering, v. 104, n. 4, p. 538-547, 2011.

LAM, P.L., GAMBARI, R. Advanced progress of microencapsulation technologies: In vivo and in vitro models for studying oral and transdermal drug deliveries, Journal of Controlled Release, v. 178, p. 25-45, 2014. 
LUPO, B. et al. Preparation of alginate microspheres by emulsification/internal gelation to encapsulate cocoa polyphenols, Food Hydrocolloids, 38, 56-65, 2014.

McCLEMENTS, D.J. Emulsion design to improve the delivery of functional lipophilic components, Ann. Rev. Food Sci. Technol, v. 1, n. 241-269, 2010.

McCLEMENTS, D.J. Selection of Delivery Systems: Case Studies In Nanoparticle- and Microparticle-based Delivery Systems, Edited by: CRC Press, pp. 401-452, 2014.

MUKAI-CORREAA, R.; PRATA, A.S.; ALVIM, I.D., GROSSO, C.R.F. Controlled release of protein from hydrocolloid gel microbeads before and after drying, Current Drug Delivery, v. 1, n. 3, p. 265-273, 2004.

MUNIN, A., EDWARDS-LÉVY, F. Encapsulation of natural polyphenolic compounds: a review, Pharmaceutics, v. 3, p. 793-829, 2011.

NEVES, M. A., HASHEMI, J., PRENTICE, C. Development of novel bioactives delivery systems by micro/nanotechnology, Current Opinion in Food Science, v. 1, p. 7-12, 2015.

O'DONNELL, P. B.; MCGINITY, J. W. Preparation of microspheres by the solvent evaporation technique, Advanced Drug Delivery Reviews, 28 (1), 25-42, 1997.

OKURO, P. K., FURTADO, G. F., SATO, A. C. K., CUNHA, R. L. Structures Design For Protection And Vehiculation Of Bioactives, Current Opinion in Food Science, Available online 18 September 2015.

PONCELET, et al. Production of alginate beads by emulsification/ internal getation. I.Methodology. Applied Microbiology and Biotechnology, v. 38, n.1, p. 39-45, 1992.

PONCELET, D. Production of alginate beads by emulsification/internal gelation. Bioartificial Organs Iii: Tissue Sourcing, Immunoisolation, and Clinical Trials, 944, pp. 74-82, 2001.
PONCELET, D., BABAK, V., DULIEU, C., PICOT, A. A physico-chemical approach to production of alginate beads by emulsificationinternal ionotropic gelation, Colloids and Surfaces A: Physicochemical and Engineering Aspects, v. 155, n. 2-3, p. 171-176, 1999.

RABE, S., KRINGS, U., BERGER, R. G. Influence of oil-in-water emulsion characteristics on initial dynamic flavour release, J Sci Food Agric, v. 83, pp. 1124-1133, 2003.

RÉ, M. I., SANTANA, M. H. A., ÁVILA, M. A. Encapsulation Technologies for Modifying Food Performance. In Taylor and Francis Group, LLC, p. 223-275, 2010.

ROCHA-SELMI, G. A., BOZZA, F. T., THOMAZINI, M., BOLINI, H. M.A., FÁVAROTRINDADE, C. S. Microencapsulation of aspartame by double emulsion followed by complex coacervation to provide protection and prolong sweetness, Food Chemistry, v. 139, n. 14, pp. 72-78, 2013.

ROCK, C.L. Carotenoids: biology and treatment, Pharmacology and Therapeutics, v. 75, n. 3, pp. 185-197, 1997.

RODRIGUES-AMAYA, D. A Guide to Carotenoid Analysis in Foods, OMNI Research: ILSI Press: Washington D. C. 1999.

SARKAR, S., GUPTA, S., VARIYAR, P. S., SHARMA, A., SINGHAL, R. S. Irradiation depolymerized guar gum as partial replacement of gum Arabic for microencapsulation of mint oil, Carbohydrate Polymers, v. 90, n. 4, p. 16851694, 2012.

SHARIPOVA, A.A., AIDAROVA, S.B., GRIGORIEV, D., MUTALIEVA, B., MADIBEKOVA, G., TLEUOVA, A., MILLER, R. Polymer-surfactant complexes for microencapsulation of vitamin $\mathrm{E}$ and its release, Colloids and Surfaces B: Biointerfaces, Available online 10 April 2015.

SHI, J., XUE, S. J., WANG, B., WANG, W., YE, X., QUEK, S. Y. Optimization of formulation and influence of environmental stresses on stability of lycopene-microemulsion, LWT - Food Science 
and Technology, v. 60, n. 2, p. 999-1008, Part 1, 2015.

TEIXEIRA, V. F. T. et al. Ion exchange kinetics of magnetic alginate ferrogel beads produced by external gelation, Carbohydrate Polymers, 111, 198-205, 2014.

UENOJO, M; MAROSTICA JUNIOR, M. R; PASTORE, G. M. Carotenóides: propriedades, aplicações e biotransformação para formação de compostos de aroma. Quím. Nova [online]. 2007, vol.30, n.3, pp. 616-622.

VOS, P., FAAS, M. M., SPASOJEVIC, M., SIKKEMA, J. Encapsulation for preservation of functionality and targeted delivery of bioactive food components, International Dairy Journal, v. 20, n. 4, p. 292-302, 2010. 